\title{
Disseminated cutaneous sporotrichosis associated with ocular lesion in an immunocompetent patient ${ }^{*}$
}

\author{
Karina Bittencourt Medeiros ${ }^{1}$ \\ Lucia Martins Diniz ${ }^{1}$
}

\author{
Luana Gomes Landeiro ${ }^{1}$ \\ Aloísio Falqueto ${ }^{1}$
}

DOI: http://dx.doi.org/10.1590/abd1806-4841.20164859

\begin{abstract}
A 59-year-old female patient, previously healthy, immunocompetent, presented left bulbar conjunctiva lesions and nodular-ulcerated lesions on the arms and cervical region, besides left cervical and retroauricular lymphadenopathy. She had previous contact with domestic cats that excoriated her face. The diagnosis was conclusive of disseminated sporotrichosis through clinical and epidemiological history and cultures of skin and ocular secretions. It evolved with good response to oral antifungal therapy.
\end{abstract}

Keywords: Eye infections, fungal; Mycoses; Sporotrichosis

A 59-year-old female patient, without any comorbidities, presented erythema, pain and burning sensation on bulbar conjunctiva (Figure 1) for 75 days. Two weeks later appeared nodules with an ulcerated center on upper limbs and cervical region, together with left cervical and retroauricular lymphadenopathy (Figure 2). After diagnostic hypothesis of sporotrichosis, laboratory tests were requested (hemogram, glucose level, hepatic and renal functions), which were all normal; anti-HIV serology, negative; thorax radiograph, without alterations. Cultures in Sabouraud agar, modified, of secretions from lesions on the arms and eyes showed cream-colored filamentous colonies that changed to brown-black (Figure 3). Microscopy of the colonies revealed thin hyphae, chambered, with daisy-shaped conidia characteristic of Sporothrix spp. The treatment was done with itraconazole $200 \mathrm{mg}$ per day for 60 days with complete symptom resolution.

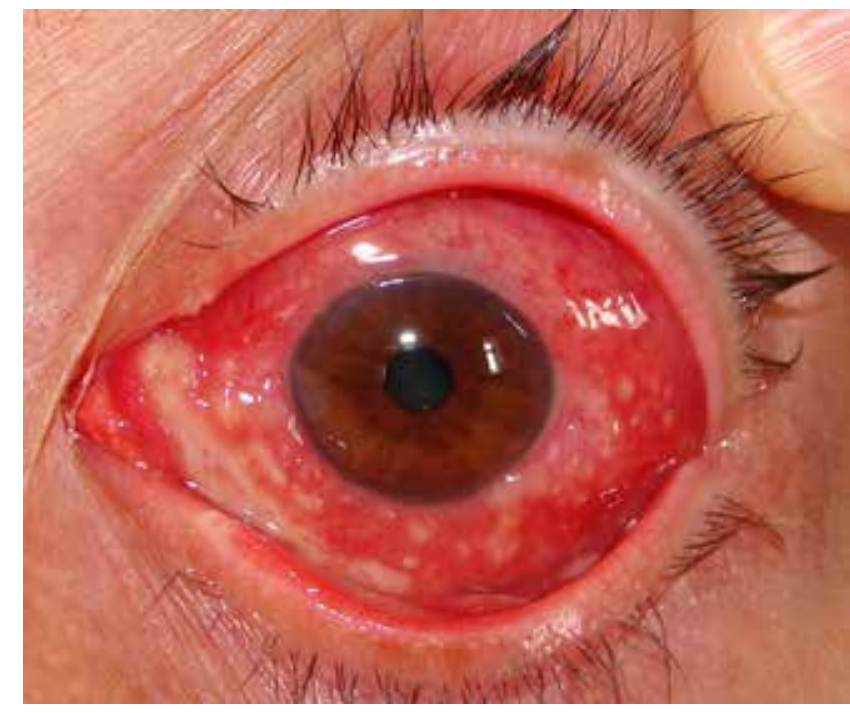

FIGURE 1 : Left bulbar conjunctiva with erythema and purulent secretion

\section{Received on 19.06.2015}

Approved by the Advisory Board and accepted for publication on 02.10.2015

Work carried out at the Dermatology Service of the Universidade Federal do Espírito Santo (UFES) - Vitória (ES), Brazil.

Financial Support: None.

Conflict of Interest: None.

1 Universidade Federal do Espírito Santo (UFES) - Vitória (ES), Brazil.

(C2016 by Anais Brasileiros de Dermatologia 

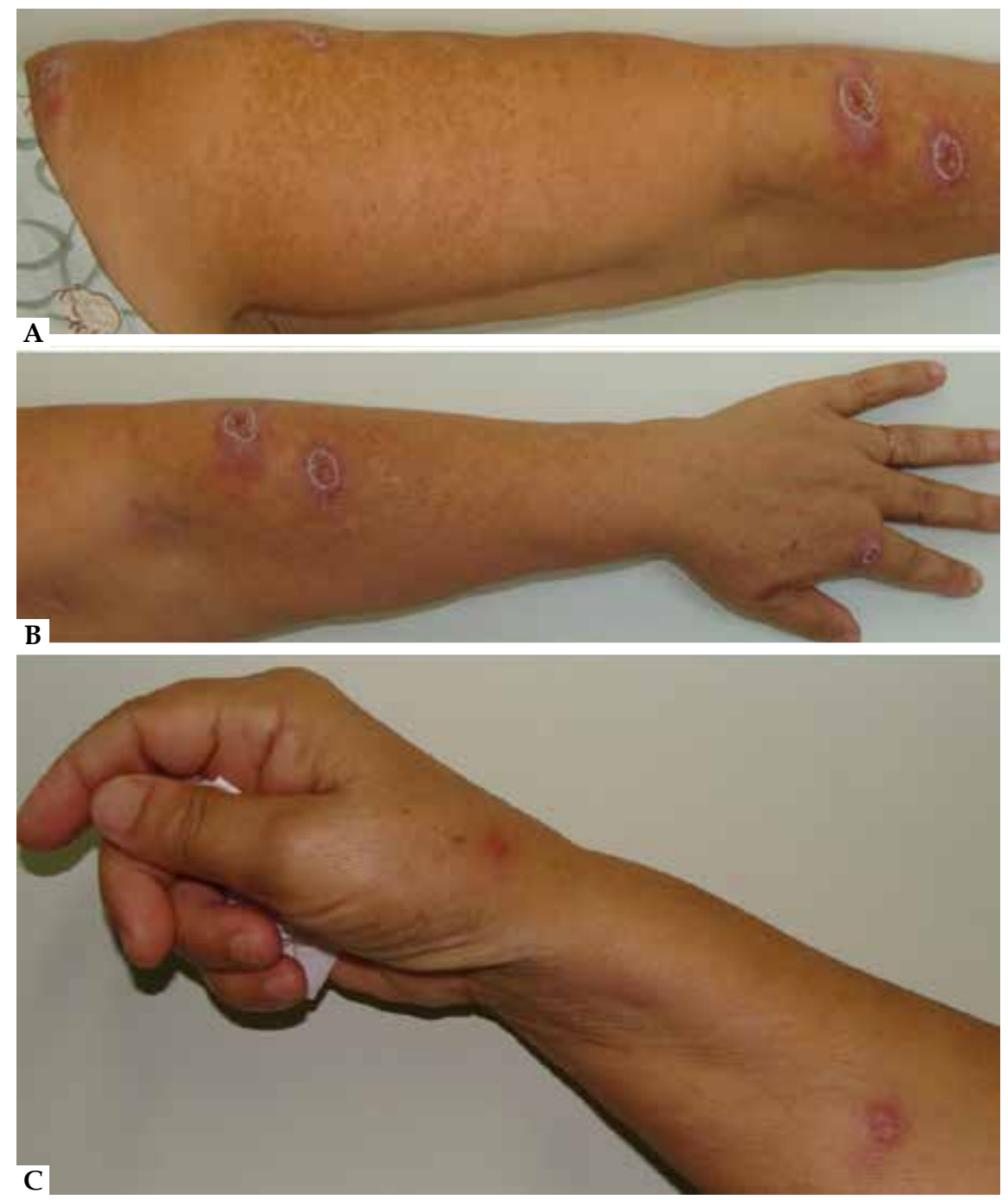

Figure 2 :

Nodular lesions with ulcerated center on left shoulder, arm and forearm (A); left forearm and back of hand (B); and right forearm and back of hand (C)

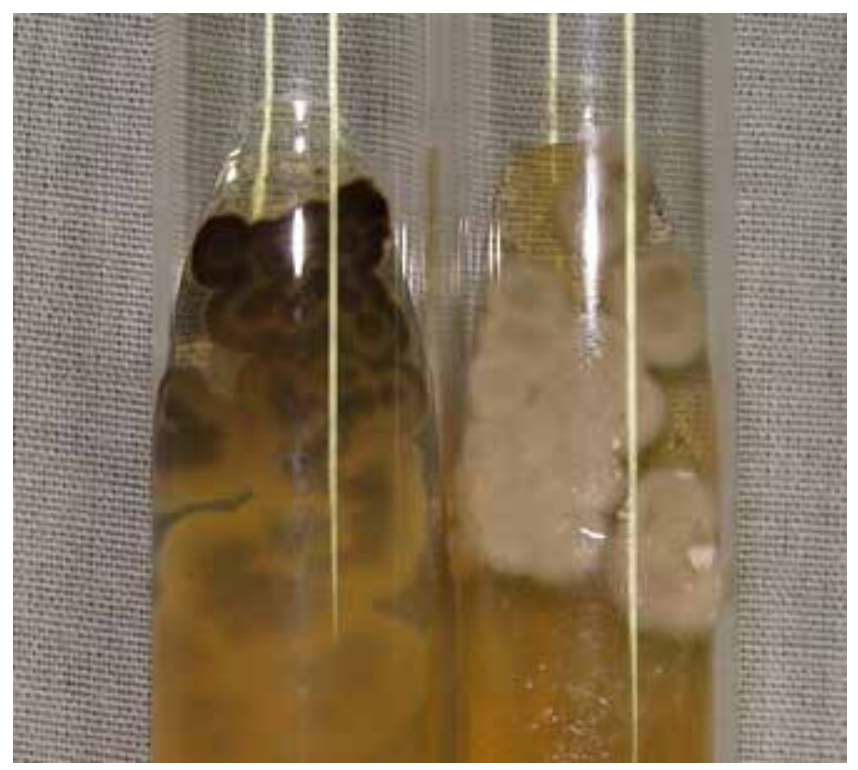

Figure 3 : Culture of eye secretion in Sabouraud Agar medium, modified, with growth of filamentous colonies, cream colored on one side and brown-black on the central portion of the other side
Sporotrichosis is a subacute or chronic disease, more common in the 30 - 60 years old; transmission may be by traumatic inoculation in the skin or subcutaneous tissue (more common) or inhalation with possible hematogenic dissemination. ${ }^{1,2}$ It is subdivided into cutaneous (lymphatic, localized and disseminated) and extracutaneous forms. ${ }^{2,3}$ The disseminated cutaneous form is rare, asymptomatic, more frequent among the immunosuppressed. ${ }^{4,5} \mathrm{~A}$ case of disseminated cutaneous sporotrichosis is here described in a woman at the expected age, with history of household contact with cats that eventually scratched her face. As the patient presented disseminated lesions, there possibly was hematogenic dissemination from the cutaneous trauma. The diagnostic was made from the clinical and epidemiologic histories associated with positive culture for Sporothrix spp. A total of 143 cases of disseminated sporotrichosis were found in global medical literature, 67 of which were in immunocompetent individuals. $]$ 


\section{REFERENCES}

1. Ramos-e-Silva M, Vasconcelos C, Carneiro S, Cestari T. Sporotrichosis. Clin Dermatol. 2007;25:181-7.

2. Morris-Jones R. Sporotrichosis. Clin Exp Dermatol. 2002;27:427-31.

3. Schubach A, de Lima Barros MB, Schubach TM, Francesconi-do-Valle AC, Gutierrez-Galhardo MC, Sued M, et al. Primary Conjunctival Sporotrichosis: two cases from a zoonotic epidemic in Rio de Janeiro, Brazil. Cornea. 2005;24:491-3.

4. Carvalho MT, de Castro AP, Baby C, Werner B, Filus Neto J, Queiroz-Telles F. Disseminated cutaneous sporotrichosis in a patient with AIDS: report of a case. Rev Soc Bras Med Trop. 2002;35:655-9.

5. Moreira JA, Freitas DF, Lamas CC. The impact of sporotrichosis in HIV infected patients: a systematic review. Infection. 2015;43:267-76.
MAILING ADDRESS:

Karina Bittencourt Medeiros

Av. Marechal Campos, 1355

Bairro Santos Dumont

29040-091 - Vitória - ES

Brazil

E-mail:karinabm18@gmail.com

How to cite this article: Medeiros KB, Landeiro LG, Diniz LM, Falqueto A. Disseminated cutaneous sporotrichosis associated with ocular lesion in an immunocompetent patient. An Bras Dermatol. 2016;91(4):537-9. 